\title{
What does the future hold for the Lung Cancer Ambition Alliance project: an interview with Giorgio Scagliotti
}

\author{
Giorgio V Scagliotti*,1 \\ ${ }^{1}$ University of Torino at San Luigi Hospital, Orbassano, Torino, Italy \\ *Author for correspondence: giorgio.scagliotti@unito.it
}

\begin{abstract}
Bio: Dr Scagliotti is currently Professor of Oncology at the University of Torino, Italy. Dr Scagliotti earned his medical degree and completed the postgraduate training in respiratory medicine, internal medicine and medical oncology at the University of Torino. He is currently chief of the Medical Oncology Division at the S. Luigi Hospital, Orbassano (Torino), former Head of the Department of Oncology at University of Torino. Dr. Scagliotti is a member of several scientific societies, including the Italian Society of Respiratory Medicine, the European Respiratory Society, the American Society of Clinical Oncology and the International Association for the Study of Lung Cancer (IASLC). From 2003-07 Executive Board Member of the IASLC. He has been Associate Editor for Journal of Thoracic Oncology and currently International Editor for Clinical Lung Cancer. He is the author or co-author of more than 360 publications in peer-reviewed journals and he is the International Editor of the 4th Edition of "Lung Cancer : Principles and practice" and co-editor of the IASLC textbook of multidisciplinary approach to thoracic Oncology.
\end{abstract}

First draft submitted: 28 September 2019; Accepted for publication: 12 November 2020; Published online: 5 February 2020

Keywords: clinical trials • lung cancer • Lung Cancer Ambition Alliance • patient engagement

\section{Questions}

Please introduce yourself to the readers \& provide a brief summary of your career to date?

I am Giorgio Scagliotti, Professor of Medical Oncology at the University of Torino and up to a couple of weeks ago, I was the President of the International Association for the Study of Lung Cancer (IASLC). I was born in Torino and then I was also graduated at the University of Torino. I moved to USA for a couple of years and then back to Italy to a smaller university close to Milano. Ten years later, I came back to Torino where I am running the Division of Medical Oncology. I did, and am still doing, a lot of clinical research focusing on thoracic malignancies.

How did you become involved in the field of lung cancer \& how did this lead to the Lung Cancer Ambition Alliance project?

The reason I became involved in the field of lung cancer is an interesting story because my residency time was in the School of Medicine, more specifically the Department of Internal Medicine. In the field of the Respiratory Medicine there were different areas and the only empty area was related to lung cancer. I started, by chance, to become interested in lung cancer. From the beginning, I realized that a lot of research in the field of the biology of lung cancer was needed. My early scientific interest was related to the way in which tobacco smoking is activated in the lung and to see if there was an opportunity to identify different categories of patients that potentially have a different risk based on exposure to tobacco smoke.

Unfortunately, those studies did not fully succeed, but they still represent the route from where I started. Eventually, I started to become more clinically involved running Phase II and Phase III clinical studies mainly in stage 4 non-small-cell lung cancer (NSCLC). In addition, I started doing a series of investigations looking into the role of systemic therapies in the field of early stage NSCLC.

This type of interest recently led me to the Lung Ambition Alliance project that started from the time I became president of the scientific organization that is the IASLC. I felt we needed an alliance involving different stakeholders

Future 8 Medicine 
that are definitively involved in the different areas of diagnosis and treatment of lung cancer. More specifically, I feel that if you want to make a significant impact on long-term survival in a reverse way, if you want to improve the 5-year survival rate with all patients, the only solution is to make diagnosis earlier.

Consequently, I suddenly realized that was the starting point to create an alliance. The alliance has four different founding members. One is the Scientific Organization that is the IASLC. The other three are the Global Lung Cancer Coalition, that is taking care of the optimization of the patient care, the AstraZeneca pharma company and Guardant Health. The role of Guardant Health is a role in perspective because I believe that molecular diagnostics will be part of the story, especially in making reasonable innovative follow-up of patients that were treated with surgery or radiotherapy after detecting the lung cancer through CT scan. The alliance as I said has four founding members with different rights, but we are totally open to other companies or to other potential stakeholders that express the will to be part of these alliances. It is an open initiative and I believe that is a pretty innovative initiative. It is something that is quite likely to be ahead of time as a concept, but I see opportunities in the near future to have other members of the alliance joining us in this kind of adventure.

\section{Please could you briefly explain the key focuses of the Lung Cancer Ambition Alliance?}

When you are making an alliance, you need to be realistic and you need to start from something. You need to have some specific goals and our primary goal is to double the 5-year survival rate in the next 10 years.

In terms of activities, we want to focus on early diagnosis and try to improve the quality of CT scan examination. In other words, we are trying to set up a system that will harmonize, as much as possible, the results of imaging across multiple centers in the world. This is just the start of the story because we need to convince heavy smokers to accept the idea of doing a CT scan and that is part of a second step - implementing the strategies, that is based on differences in country regulations. That is the strategy and that is what we are currently initiating in addition to the quality control that I was mentioning before.

Now we are starting to think about how to become strategic in the implementation country by country, how we can convince heavy smokers to accept the idea to do the imaging testing. There are additional data that were presented at the World Lung conference that shows us that microRNA can improve the success rate in detecting renal cancers.

The second series of initiatives is related to getting the patient innovative medicine as soon as possible and that leads me to the concept of neoadjuvant chemotherapy. I believe that we need to learn how to use systemic therapies or how to implement systemic therapies in the treatment of early stage NSCLC. At the same time, we need to learn how to implement local therapies in the management of stage 4 NSCLC.

Coming back to the first point, I truly believe that the integration of screening, surgery or radiotherapy and the implementation of systemic therapies will be the key to significant improving long-term survival. If you do an adjuvant trial, it takes a lifetime to get the results. If you do a neoadjuvant study, you are getting a lot of information about the molecular characteristics of the tumor because you are getting baseline information.

At the time of surgery, you are getting a second tissue sample and based on the changes you see in the molecular landscape, you will be able to modulate the adjuvant therapy.

The last pillar is related to the optimization of lung cancer patient care and this is exactly where the Global Lung Cancer Coalition is coming into the field. We also have initiatives in this field and one of these initiatives will be launched by the Global Lung Cancer Coalition ESMO 2019 today.

Finally, there is a fourth pillar that is transversal to the first three, that is related to smoking cessation. Smoking cessation is critical, and the implementation of smoking cessation strategies is another piece of the story that is critical to be included in the initiative.

\section{You have touched on this, but what do you think the future holds for the Lung Cancer Ambition Alliance \& what challenges do you think may be faced?}

The future is based on the present so we are currently working on the present and we would like to succeed with all the initiatives that I have summarized for you. I believe that the future will be highly dictated by the metrics of success that we will be able to achieve. The future stands in the implementation of the activities that I was mentioning before in each individual country, because the value of the alliance is based on a different level of expertise that are united by the common goal of defeating lung cancer. There are challenges because you need to buy an idea and you need to transfer the same level of enthusiasm to the community treating lung cancer that the four founding members have. Only with the direct involvement of different people at different levels with 
connection to the communities would we be able to do initiatives that would really impact the future of the Lung Ambition Alliance.

Why do you think it is important to engage patients in research \& clinical studies?

It is impossible to succeed in any trial if you are not making patients aware of what you are doing. Patients are not passive subjects. You need to explain the value of your diagnostic and therapeutic interventions.

For example, if you are considering the overall population that should be considered at risk for lung cancer (the main population above 55 years of age, or a current smoker and smoking more than one pack of cigarettes a day), only $5 \%$ of this population, even less, are receiving a low dose CT scan for early diagnosis. If we are able to convince 15 or $20 \%$ of the high risk group to do a CT scan, we will be able to detect $80 \%$ of lung cancer cases in stage 1 and stage 2 , and we will be able to impact significantly the 5 -year mortality.

\section{Finally, how would the Lung Cancer Ambition Alliance help to engage patients in this way?}

You need to convince them through communication strategies that there are tools to diagnose lung cancer earlier. We need to involve the Ministry of Health in each country because any project comes with investments. Investments are now possible because, for example, 10 years ago the reading of a CT scan was totally human based and that means a huge investment in terms of human resources. Now we are really at the sunrise of artificial intelligence tools and $90 \%$ of these scans can be read by software and consequently the amount of work that stays in the hands of a radiologist is only $10 \%$. But again, this is a moving target and a few years from now, we will have new tools that will make our lives easier.

The alliance is a dynamic constant framework, that probably will reshape itself every 2 years and it is important that in each country high risk patients are involved. They need to be aware that this is a nonprofit organization in which different players from pharma companies, from diagnostic companies, from nonprofit organizations which are placing the patients at the center of the stage and they want to get the critical mass able to significantly impact 5 -year survival rate for lung cancer.

\section{Disclaimer}

The opinions expressed in this interview are those of the interviewee and do not necessarily reflect the views of Future Medicine Ltd.

Financial \& competing interests disclosure

GV Scagliotti received Honoraria from Roche, Astrazeneca, MSD, Celgene, Pfizer, Takeda, Eli Lilly. The authors have no other relevant affiliations or financial involvement with any organization or entity with a financial interest in or financial conflict with the subject matter or materials discussed in the manuscript.

No writing assistance was utilized in the production of this manuscript. 
